\title{
Classification of Mental Disorders Figures based on Soft Computing Methods
}

\author{
Jabar H. Yousif \\ Faculty of computing \& IT \\ Sohar University \\ P.O.Box. 44, P.C. 311, Sohar, Sultanate of Oman
}

\begin{abstract}
The purpose of this paper is to present the design and implementation of Soft Computing approaches (SOFM and MLP) for classifying mental disorders figures. World Health Organization (WHO) referred to the higher rates of mental disorders as a result of the pressures of life and its difficulties. This prompted the non-governmental organizations and civil institutions to increase research and reduce the risk of mental illness. However, despite all these efforts, the researchers still did not achieved the anticipated results. The classification of mental disorders is a significant concern for researchers as well as health care service providers. The comparison study is tested two different techniques under the same environment, conditions and using same data. The MLP achieved high accuracy of (99\%), while SOFM achieved an accuracy of (97\%) in the classification phase for predicting the desired output. In the meaning of data amount usage, both MLP \& SOMF are used a small amount of data to perform the training phase. While other approaches like SVM are huge data sets to perform the training phase. Consequently, using a few data in the learning network phase leads to a rapid process and getting a results in short time.
\end{abstract}

\section{General Terms}

Computer science, artificial intelligence, neural network, applications, soft computing.

\section{Keywords}

Mental illness, MLP, SOFM, text clustering, text classification, unsupervised earning.

\section{INTRODUCTION}

The mental disorders can be defined generally through a combination of features that reflect the feelings of a person or his actions and explain his thinking and perceptions. It is concomitant with many chronic conditions such as high blood diabetes, pressure, HIV / AIDS, resulting in additional costs to the health system. Understanding of the causes and types of mental illnesses may help to improve individual experiences in order to develop solutions or to diagnose the truth causes of mental disorders. Mental illnesses include psychological or behavioral configurations that are frequently correlated with distress or disability $[4,8]$. The significant growth in data transfer and rapid rise in health care costs the needs extensive studies to achieve an efficient and inexpensive solutions in classification, data analysis and data extraction $[13,16,19]$.

The main source of the difficulty in classifying mental disorders is incomplete understanding of their causes and figures. The implementation of artificial intelligence techniques in health care problems can help to diagnose diseases and assess treatment outcomes. Soft computing (SC) is referring to the approaches of calculation that discover approximate solutions for satisfying real-world problems. The SC relies on the use of the neural networks, fuzzy logic, and support vector machines. The main objective of $\mathrm{SC}$ is simulating human capacities such as learning, thinking and problem solving, etc. Recent developments in the field of information technology and the use of soft computing techniques have helped to develop effective automated diagnostic systems [14, 20,23].

The using the artificial neural network is consider as a most qualified methods for learning from the rare data. The purpose of this paper is to design and implement a text classification model for identifying mental illness using a multilayer perceptron neural network (MLP) and Self-Organizing Map (SOM). The ANN have been implemented successfully in various fields such as image processing, NLP, speech recognition, pattern recognition and classification tasks [3,15].

Professional psychiatrists work hard consciously and unconsciously to investigate the language of their patients with the intention of identifying patterns, and use these patterns to assist in building an obvious clinical diagnosis [10, 23]. Clustering is the process of separating data into groups that have similar features. Clustering is one of the significant themes of research that demonstrates important successes in many fields of research such as statistics, pattern recognition and machine learning [13, 17]. All learning techniques required automated input samples to complete the training phase. And naturally the learning processes need to use a large number of samples. On the other hand, the causes of some mental illnesses are not recognized till now. Therefore, it is difficult to obtain models for experiments. $[7,8]$.

\section{MENTAL HEALTH BACKGROUND}

The Medilexicon's medical dictionary is defined the mental illness as "emotional, behavioral, and social maturity or normality; a state of psychological well-being in,, which one has achieved a satisfactory integration of one's instinctual drives acceptable to both oneself and one's social milieu; an appropriate balance of love, work, and leisure pursuits"[21]. The health care of mental illness is imperative and it should be available in every stage of life since childhood and through puberty. The term "mental illness" generally includes various type of mental disorders, which are well categorized by the health care specialized. Today, mental illness is become one of the main problems in our society [2]. The World Health Organization (WHO) expects the world's leading disability will be one of the most important problems in the world and mainly caused by depression by 2020 .

The Medilexicon dictionary defines the depression as "a mental state or chronic mental disorder characterized by feelings of sadness, loneliness, despair, low self-esteem, and self-reproach. All ages vulnerable to Mental illness, but young and old are most vulnerable ages for a particular disease to lack of experience in life, and lack of knowledge of the ways to deal with the pressures of life. Different services provided to people with mental health problems from one country to another and depend on the ratio of government support for the 
sector through programs funded health care and the number of public and private hospitals for treatment of mental health patients and caregivers [5].

There is an urgent need for the establishment of a range of community services,, which assist in the follow-up and reduce the risk of psychiatric association such as clinic-based, mobile follow-up and treatment, could set up an association to follow patients and communicate with patients and provide advice and guidance. Despite important achievements and significant in the field of mental health in some countries over the past decade, but there is still much work needed for addressing the mental health problems. As a result of increasing numbers of people with mental illness, there became a need to increase the research and studies in the field of mental health. Research covered a wide range of information that describes the different types of mental illnesses. In order to find effective solutions, which reduce the risk of these diseases $[9,12]$.

\section{ANN BACKGROUND}

Artificial Neural network (ANN) is a data modeling tool that is able to capture and simulate the behavior of the human brainpower. The neural networks a powerful tool capable of representing complex inputs both linear and Non-linear relationships [18]. There are certain advantages prompted scientists to adopt the using of neural networks to solve problems in different application areas. These features include parallelism, uniformity, and representation \& distribution. Besides, it has the ability to learn, train, adaptation and generalization [15].

ANN had been positively implemented in different areas like data classifications and retrieving, resource scheduling and allocation, data analysis and mining, speech recognition and production, and pattern recognition and generation, etc. [18]. The Self-Organizing Feature Map (SOFM) is one of the popular ANN techniques that train by using un-supervised learning to transform a multi-dimensional input space into a low-dimensional output space. A number of developed projects are using the SOFM for solving solid real- problems [15]. The SOFM had been implemented in a number of field's science as an analytical tool such as signal processing, control theory, financial data analyses, physics, chemistry and medicine experiments.

\section{RELATED WORK}

Imianvan a.a. \& obi j.c. [13] a classical procedure for the medical diagnosis of Bipolar disorder is proposed by Imianvan. The medical diagnosis is employed by physician doctors initially and Imianvan proposed to analysis it using Neuro-fuzzy technique. The Neuro-Fuzzy Logic adopts approximation procedures based neural networks to find the suitable parameters of a fuzzy system. Jabar \& Mabruk [14] A Multilayered Perceptron (MLP) is designed and implemented to categorized a transcribed speech samples and discover the mental health disorders. The proposed system is used a classification techniques to decide if an input text sample is produced by somebody who has a mental health problem like schizophrenia or mania. The classifications of schizophrenia are wide-ranging. Thus, the clustering techniques are used to achieve the task-relevant diagnosis. The demonstrating of proposed system is achieved a high accuracy of (99\%). C. R. S. Lopes \& et. al. [10] A Multilayer
Percepton (MLP) is used to analyze common mental disorders (CMD) factors trained based on the Simulated Annealing Algorithm (SAA). The architectures and weights of the system are optimized using the SAA simultaneously. It is aims to identified variables, which occur with higher frequency with the mental disorder being studied. The experiments on neural networks showed that the years of schooling were more often associated with CMD with 89.29\%. Mabruk and Joachim [1] implemented a text mining techniques to determine psychiatric problems using the clustering and classifications techniques. They aims to identify speech samples either was generated by person have mental illness or not. The research achieved high accuracy $(92 \%)$ and precision $(87 \%)$ and moderate to low recall. Gal Kazas \& Michael Margaliot [11] a Self-Organizing Feature Map (SOFM) based algorithmic approach is proposed for visualizing the topology of mental configurations. The SOFM is trained to create a two-dimensional feature map of 27 recognized mental conditions. Originally, an 82-dimensional input vector is used for describing the symptoms associated with each disorder. Each input vector must be represented a unique cluster of mental disorders, which provides a method to visualize the mental disorders configures clearly.

\section{SOFM CONFIGURATION AND DESIGN}

The Kohonen's Self-Organizing Feature Map (SOFM) is consider as a data visualization system that maps a high dimensional input vector and producing a low- dimensional output vector (one or two dimensions) [15, 22]. The reducing the dimensionality of vectors is known as vector quantization,, which is an essential data compression technique. Furthermore, the Kohonen technique is used to create a network that stores information of topological relationships within the training set. SOFM is the representation clustering of data by grouping similar sets together. Thus the SOFM can reduce the number of data dimensions and produce a relation of similarities among the data. SOFM is a type of competitive neural networks that has a 2-dimensional featured space organizing neurons. The transformation of data is based on the learning rule vectors, which mean the multidimensional space must represent the similar data behavior in the 2dimensional space. SOFM are frequently used to visualize and present n-dimensional space.

Teuvo Kohonen said [22]: "The SOM is an effective software tool for the visualization of multi-dimensional information. It helps to map complex nonlinear relationships with highdimensional input vector into simple geometric relationships of low-dimensional data. It compresses the information while preserving the most significant features and metric relationships to visualize. A sample vector is calculated for all weight vectors for recognizing the greatest matching component,, which implement through and distance each weight. The winner weight is the shortest distance. The Euclidean Distance is the most common way to determine the distance between weights. In the second layer's, each neurons have dimension equal to the dimension of the input layer. The architecture of SOFM is depicted in Fig 1. The SOFM Neural Network consists of two layers. The input layer,, which receives the input data vectors and then transfers them to next layer. The neighborhood relation is implemented to connect adjacent neurons as depicted in Fig. 1 


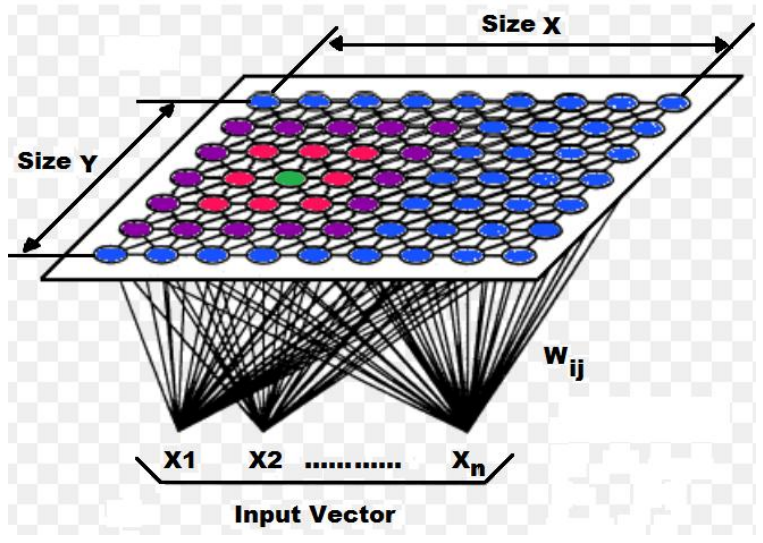

Fig.1: The architecture of SOFM

The learning rule is the study of adaptable data nodes and generalized the output nodes based on historical learning examples. First initializing each node's in weights vector and set all weights vectors of the second layer's neurons to random values. Then choose randomly a vector from the set of the training data and determine the Best Matching Unit (BMU): $\quad D_{i j}=\min _{\mathrm{i}, \mathrm{j}}\left(\left\|X_{i}-W_{i j}\right\|^{2}\right)$

Different methods are used to determine the neighbors of a winning weight like Concentric Squares, Hexagons, Gaussian, Hamming, Mexican hat, French hat, etc. In real applications, the Mexican hat $(h(\rho, t))$ or the French hat $(h(\rho))$ is used. The weight vector is updated only for winner neuron or adjacent to the winner. On each step, the $\mathrm{NN}$ determines the neuron whose weights vector is the best similar to the input vector. The current weights vectors and its neighbors' are updated corresponding to input vector as depicted in Fig 2.

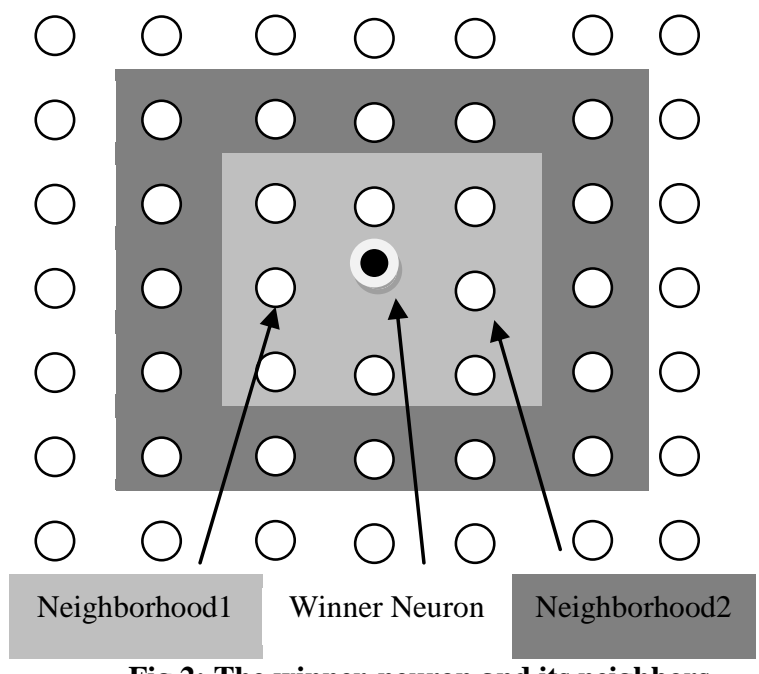

Fig.2: The winner-neuron and its neighbors

\section{MLP CONFIGURATION \& DESIGN}

The multilayer perceptron (MLP) is one neural network topologies, which is widely implemented to adapt and solve most of the problems in real-life. The function of MLP can adopt most shapes of input data clusters. The MLP can achieve the maximum performance, which is equivalent to optimal classification form, if the weights and the output classes are properly normalized to 0/1, [18]. A MLP network can be learned using different learning techniques. The error back-propagation algorithm is widely used as learning techniques,, which propagates the errors and allows adaptation of the hidden layers for simulating the desired behavior. [15]. It can be implemented using some examples of desired input/output data sets to the network. A correction of weights is performed frequently so that the network is stable. Then the network is learned to produce the desired output. The error correction learning $\operatorname{err}_{\mathrm{i}}(\mathrm{n})$ is defined as follows:

$$
\operatorname{err}_{\mathrm{i}}(\mathbf{n})=\mathrm{d}_{\mathrm{i}}(\mathbf{n})-y_{i}(n)
$$

yi(n) is the output from the perceptron for an input vector i, and the preferred output. The $\operatorname{di}(\mathrm{n})$ is the value of perceptron for a given input pattern. The gradient descent learning method is used to adapt each weight in the network as follows:

$$
\mathrm{w}_{\mathrm{ij}}(\mathrm{n}+1)=\mathrm{w}_{\mathrm{ij}}(\mathrm{n})+\eta \delta_{i}(n)+x_{j}(n)
$$

The local error $\operatorname{err}_{\mathrm{i}}(\mathrm{n})$ is computed as a sum of errors at the interior PEs. The constant momentum step size is $\eta$ and

Momentum learning factor is $\delta_{i}(n)$. It is used to speed up and stabilize convergence by update the weights of network based on some of the last steps error adjustments. It illustrated as follows:

$$
\begin{gathered}
\mathrm{w}_{\mathrm{ij}}(\mathrm{n}+1)=\mathrm{w}_{\mathrm{ij}}(\mathrm{n})+\eta \delta_{i}(n)+x_{j}(n)+ \\
\alpha\left(\mathrm{w}_{\mathrm{ij}}(\mathbf{n})-\mathrm{w}_{\mathrm{ij}}(\mathbf{n}-\mathbf{1})\right)
\end{gathered}
$$

The $\boldsymbol{\alpha}$ is the momentum value. Typically is to set its value between 0.1 and 0.9 , the best value is 0.7 .

This paper presents a design and implementation of a MLP as demonstrated in Fig. 3. The network consist of one hidden layer, and 11 input Processing Elements (I1,I2,..,I11), while it comprises of 6 output Processing Elements $(\mathrm{O} 1, \mathrm{O} 2, \ldots, \mathrm{O} 6)$. The maximum numbers of epochs in this experiment are 1000. The TanhAxon is used as transfer function in hidden and output layers. This will squash the range of each neuron in the layer to value between (-1) and (1). However, in sake of giving the network the ability to construct soft decisions, the nonlinear elements are used. The momentum learning rule is adopted with constant step size equal to (1), and momentum rate equal to $(0.7)$.

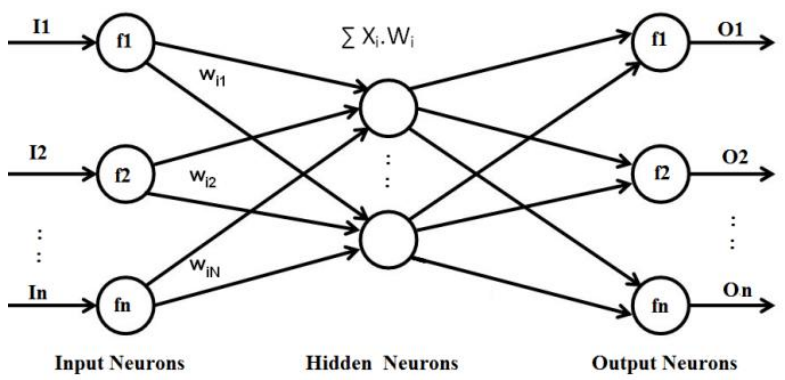

Fig.3: The architecture of MLP

\section{EXPERIMENTS AND RESULTS}

For the purpose of testing the proposed two networks architectures, two hundred messages were selected randomly from each "sci.psychology psychotherapy" and "sci.philosophy". The header and signature information is removed from the text messages for the sake of unrecognizability of messages contain $[1,16]$. The experiments used 200 text files comprising the original message plus their replies. Further a pre-processing is 
employed using the text samples to encode them into binary values, which are suitable form for the neural network. However, the inputs of the network are transcribed speech samples in binary forms. And, the output will determine whether the text has been written by someone who has mental illness. This is done by searching the text for key symptoms of mental illness such as attention, deficit, hyperactivity, and disorder (ADHD) $[1,16,17]$. In this paper the experiments determined nine clusters (ADHD, attention deficit, disorder, hyperactivity, apprehensive, deranged, psychotherapy and none of them)

The data set is separated into three sets, first is the training data set, which is used to train the network and produce the best neuron weight to generalize the network. The experiments used about 500 data sets for the training process. The second data set is the cross validation,, which is estimated the accuracy of test data set at the time of training the network. The experiments used about 400 data sets for cross validation process. And the third set is testing data to determine the best network performance. The experiments used about 71 data sets for the testing of network performance. The weights of best network are automatically saved during the training phase and will be loaded into the network before the testing process is come over.

The experiment of neural networks (SOFM) is performed using the NeuroSolutions software package. The unsupervised (Kohonen) layer is implemented to deploy the shape and size of the feature map. Besides, it determines the number of Hidden Layers in the network of the MLP connected to its output. One Hidden Layer was used and the size and dimension of the unsupervised output space are 5 Rows and 5 Columns of the 2D neural field. Increasing the number of Process Elements (PEs) will improve the resolution, but also amplify the training times. The experiments used 12 and 9 PEs for input and output respectively. Basically, the size of the Kohonen layer must be maintained as small as possible because the SOFM trains slowly.

The Starting and Final Radius are 2 and 0 respectively. The Back propagation is a well-known approach of learning techniques that helps to reduce the value of weights. The momentum component is used as learning rule. The step size is 1 and the momentum rate is 0.7 . The Genetic optimization is used to find the best values for these parameters. The networks performance is measured based on many factors [6]. Usually, the mean squared error MSE is used. It is computed as follows:

$$
M S E=\frac{\sum_{j=0}^{P} \sum_{i=0}^{N}\left(d_{i j}-y_{i j}\right)^{2}}{N P}
$$

The number of output processing elements is $\mathrm{P}$. The number of training set is $\mathrm{N}$ is and the network output is $\mathrm{y}_{\mathrm{ij}}$ for data set $i$ at processing element $j$. The $d_{i j}$ is the desired output for network. The outputs of the SOFM network for three runs are summarized in Tables 1,, which records the minimum and final MSE. The Figs 4-A and 4-B depict the graphs of best network of training and cross validation data respectively for SOFM network. And it is achieving an accuracy of (97\%) in classification task and predicting the desired output. The outputs of the MLP network for three runs are summarized in Tables 2, which records the minimum and final MSE. The Fig. 5 depicts the graphs of best network of training for the MLP network, which is achieving a high accuracy of (99\%) in classification task and predicting the desired output.
Table 1. Minimum and final MSE of SOFM

\begin{tabular}{|c|c|c|}
\hline Best Networks & Training & Cross Validation \\
\hline Run \# & 2 & 2 \\
\hline Epoch \# & 999 & 999 \\
\hline Min. MSE & 0.008409 & 0.008080 \\
\hline
\end{tabular}

Table 2. Minimum and final MSE of MLP

\begin{tabular}{|c|c|c|}
\hline Best Networks & Training & Cross Validation \\
\hline Run \# & 2 & 2 \\
\hline Epoch \# & 1000 & 1000 \\
\hline Min. MSE & 0.006025 & 0.006096 \\
\hline
\end{tabular}

\section{CONCLUSION}

The current work used the same data sets,, which are used in related studies $[1,16]$ for both MLP \& SOFM networks. This is only to test the experiments in same environment and under the same conditions. The results of the comparison of this work with other researcher related mental health studies [1, $14,16,17,20]$ are summarized in Table 5 ,, which give an evidence the proposed work has high accuracy ( $99 \%$ and $97 \%$ ). Besides, it used a small amount of data to for the training phase. While Fig 6 shows the comparison result of MSE for training phase of MLP \& SOFM, which it is clearly illustrated the training phase of SOFM is better than MLP. Besides, the mean squared error MSE of SOFM is less than MLP that as result of using the representation clustering of data by grouping similar sets together. Thus the SOFM can reduce the number of data dimensions and produce a relation of similarities among the data faster than the original data that used in MLP. On other hand, the MLP achieve high accuracy in classification of the input text, while the SOFM fail to identify the type O8 (psychotherapy). Nine sings of mental illness are analyzed and implemented ( O1 is ADHD, O2 is attention $\mathrm{O} 3$ is deficit, $\mathrm{O} 4$ is disorder, $\mathrm{O} 5$ is hyperactivity, O6 is apprehensive, $\mathrm{O} 7$ is deranged, $\mathrm{O} 8$ is psychotherapy and $\mathrm{O} 9$ is none of them).

Nevertheless, the robustness of the machine learning classification and clustering is implemented in this paper. It has been shown that the MLP is demonstrated a high accuracy of $(99 \%)$. As well the SOFM network had achieved a high accuracy of $(97 \%)$ in classification of the input text. Both approaches are predicted if it is a type of mental illness or not. Besides, the proposed approaches use a small number of data to complete the classification process and train the network,, which is useful in experiments with rare data.

\section{FUTURE WORK}

The experiments proved more attention must devote to track the history record of patients. It may give key features,, which identifying the illness's causes. Besides, the genetic algorithms can be used to enhance the results especially in clustering phase. The automatic pre-processing phase including the reading, encoding, and clustering of data is needed to reduce human errors is required. Furthermore, implementation of other cloud computing techniques such data mining analysis can give more clarification about the mental illness causes. 


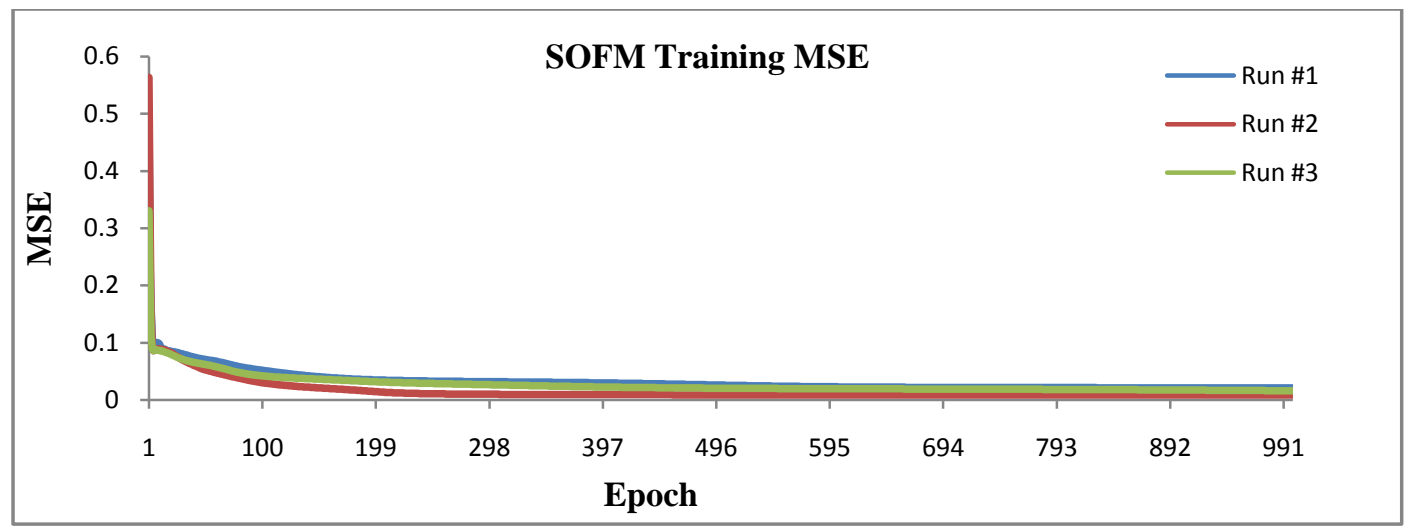

Fig.4-A: The results of MSE for training phase of SOMF network

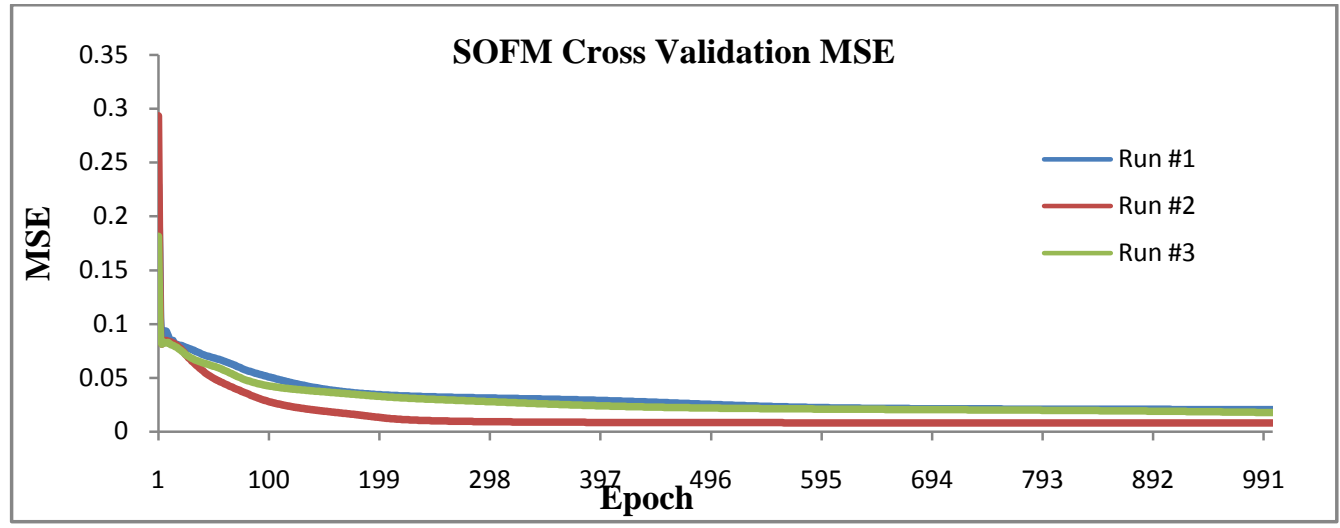

Fig.4-B: The results of MSE for cross validation phase of SOMF network

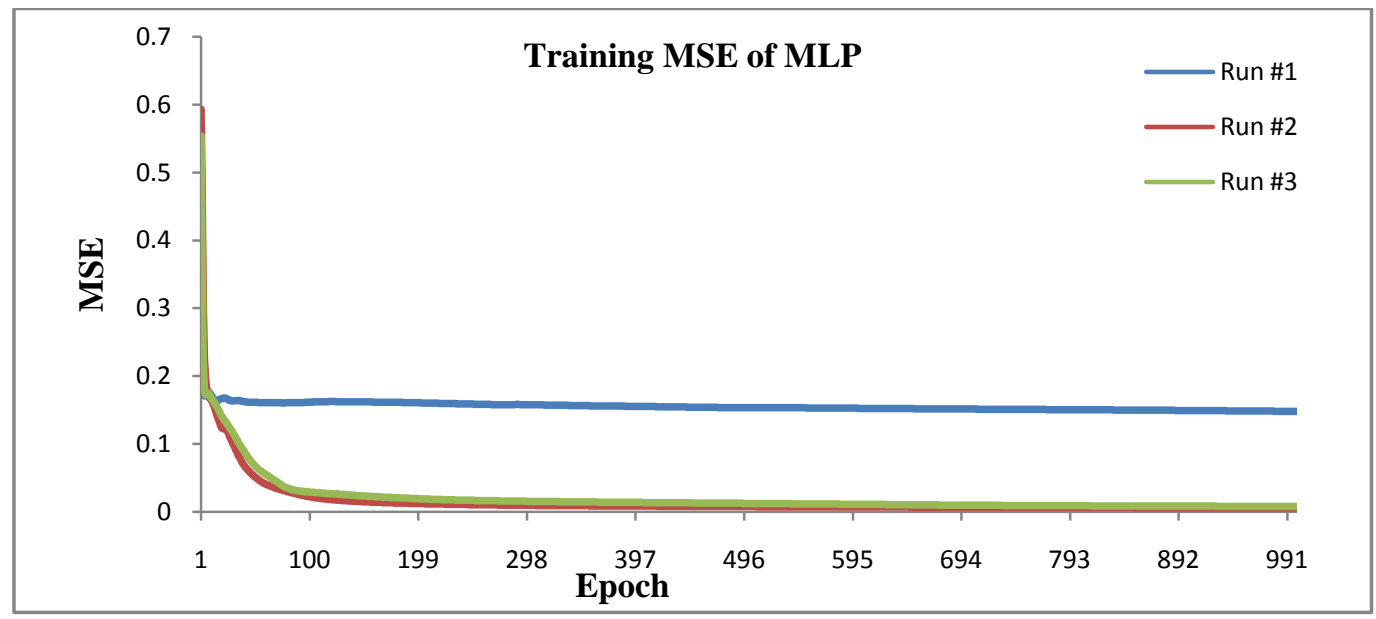

Fig. 5:The results of MSE for training phase of MLP network

Table 3: the results of resting MLP

\begin{tabular}{|c|c|c|c|c|c|c|c|c|c|}
\hline Performance & O1 & O2 & O3 & O4 & O5 & O6 & O7 & O8 & O9 \\
\hline MSE & 0.0033 & 0.0046 & 0.0156 & 0.0049 & 0.0003 & 0.0028 & 0.0018 & 0.0022 & 0.0174 \\
\hline NMSE & 0.0819 & 0.1125 & 0.3839 & 0.0915 & 0.0247 & $\#$ DIV/0 & 0.1263 & 0.1608 & 0.0997 \\
\hline MAE & 0.0392 & 0.0489 & 0.0527 & 0.0489 & 0.0108 & 0.0460 & 0.0362 & 0.0338 & 0.0617 \\
\hline Min Abs Error & 0.0001 & 0.0004 & 0.0004 & 0.0014 & 0.0000 & 0.0003 & 0.0011 & 0.0004 & 0.0023 \\
\hline Max Abs Error & 0.3350 & 0.2394 & 0.9784 & 0.4331 & 0.1141 & 0.2034 & 0.1197 & 0.22762 & 0.98022 \\
\hline r & 0.9651 & 0.9505 & 0.8517 & 0.9658 & 0.9882 & \#DIV/0 & 0.9551 & 0.9337 & 0.9531 \\
\hline Percent Correct & 100 & 100 & 100 & 100 & 100 & \#N/A & 100 & 100 & 98.1818 \\
\hline
\end{tabular}


Table 4: The results of resting SOFM

\begin{tabular}{|c|c|c|c|c|c|c|c|c|c|}
\hline Performance & O1 & O2 & O3 & O4 & O5 & O6 & O7 & O8 & O9 \\
\hline MSE & 0.0002 & 0.0003 & 0.0126 & 0.0002 & 0.0071 & 0.0001 & 0.0000 & 0.0071 & 0.0127 \\
\hline NMSE & 0.0062 & 0.0086 & 0.3121 & 0.0033 & 0.5111 & $\#$ DIV/0! & 0.0026 & 0.5120 & 0.0727 \\
\hline MAE & 0.0080 & 0.0129 & 0.0223 & 0.0066 & 0.0173 & 0.0039 & 0.0038 & 0.0167 & 0.0201 \\
\hline Min Abs Error & 0.0002 & 0.0036 & 0.0003 & 0.0003 & 0.0002 & 0.0000 & 0.0000 & 0.0000 & 0.0001 \\
\hline Max Abs Error & 0.0552 & 0.0554 & 0.9342 & 0.0547 & 0.5037 & 0.0551 & 0.0177 & 0.5520 & 0.9399 \\
\hline r & 0.9972 & 0.9973 & 0.8584 & 0.9986 & 0.6992 & \#DIV/0! & 0.9989 & 0.7026 & 0.9638 \\
\hline Percent Correct & 100 & 100 & 100 & 100 & 100 & \#N/A & 100 & 0 & 98.18181818 \\
\hline
\end{tabular}

Table 5 Comparison results of proposed approach with other researchers

\begin{tabular}{|l|c|c|c|c|c|}
\hline & $\begin{array}{c}\text { C. R. S. Lopes } \\
\text { \& et. al. [10] }\end{array}$ & Joachim [16] & Abusaa, M [1] & Proposed MLP & Proposed SOFM \\
\hline Technique implemented & NN-( MLP) & (SVM) & (SVM) & NN-( MLP ) & NN-(SOFM) \\
\hline $\begin{array}{l}\text { Data sets size for training } \\
\text { phase }\end{array}$ & $100 \%$ & $100 \%$ & $100 \%$ & $20 \%$ & $20 \%$ \\
\hline Accuracy of classification & $89.29 \%$ & $80 \%$ & $92 \%$ & $99 \%$ & $97 \%$ \\
\hline
\end{tabular}

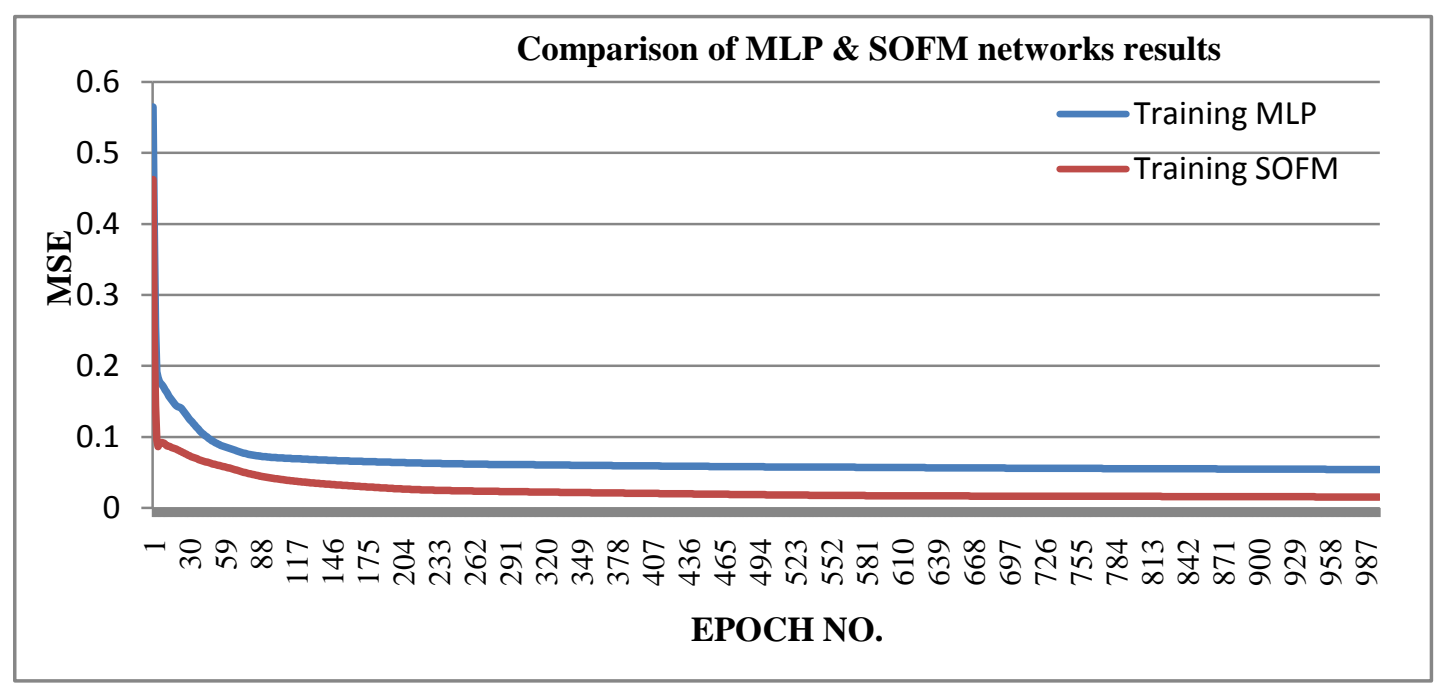

Fig. 6: Comparisons of MLP \& SOFM

\section{REFERENCES}

[1] Abusaa, M., Diederich, J. and Al Ajmi, A. (2004). Web mining and mental health. In: IAWTIC 2004 Proceedings. International Conference on Intelligent Agents, Web Technologies and Internet Commerce, Gold Coast, Queensland. 12-14 July 2004.

[2] Adelman, H. \& Taylor, L. (2006). The current status of mental health in schools: A policy and practice analysis. Los Angeles, CA: Center for Mental Health in Schools at UCLA. http://smhp.psych.ucla.edu/currentstatusmh.htm

[3] Akyol, D. E. (2004). Applications of neural networks to heuristic scheduling algorithms. Computers and Industrial Engineering, 46, 679-696.

[4] Armstrong, R., et.al,(2008). , Systematic reviews in public health, International Encyclopedia of Public Health, 297-301.
[5] Bauer, S., Percevic, R., Okon, E., Meerman, R., \& Kordy, H. (2003). Use of text messaging in the aftercare of patients with bulimia nervosa. European Eating Disorders Review, 11, 279-290.

[6] Beyer, H.-G., \&Schwefel, H.-P. (2002). Evolution strategies: A comprehensive introduction. Natural Computing: An International Journal, 1(1), 3-52. DOI: 10.1023/A:1015059928466

[7] Bonissone, P (2002). Hybrid Soft Computing for Classification and Prediction Applications. Conferencia Invitada. 1st International Conference on Computing in an Imperfect World (Soft-Ware 2002), Belfast. DOI: 10.1007/3-540-46019-5_28.

[8] Chang, W. C., et al. (2010), Factors affecting the use of health examinations by the elderly in Taiwan, Archives of Gerontology and Geriatrics, 50(1), S11-S16. 
[9] Clarke, G., Lynch, F., Spofford, M., \& DeBar ～, L. (2006). Trends influencing future delivery of mental health services in large healthcare systems. Clinical Psychology: Science and Practice, 13 (3) , 287-292. doi:10.1111/j.1468-2850.2006.00040.

[10] C. R. S. Lopes, et.al. (2002) "Neural Networks for the analysis of Common Mental Disorders Factors." Proc. of the VII Brazilian Symposium on Neural Networks (SBRN'02) IEEE Computer Society Washington, DC, USA @2002 , ISBN:0-7695-1709-9

[11] Gal Kazas \& Michael Margaliot, "Visualizing the topology of mental disorders using selforganizing feature maps". http://www.eng.tau.ac.il/ michaelm/kazas.pdf

[12] Hadzic, M., Chen, M., \& Dillon ,T. (2008) . Towards the mental health ontology, proceeding of the IEEE International Conf. on Bioinformatics and Biomedicine, USA. DOI: 10.1109/BIBM.2008.59

[13] Imianvan A.A. and Obi J.C. (2011): Diagnostic evaluation of Hepatitis utilizing Fuzzy Clustering Means, World Journal of Applied Science and Technology, Vol.3. No.1 23-3.

[14] Jabar H.yousif, Mabruk a. Fekihal , (2012), Neural Approach For Determining Mental Health Problems , Journal of Computing, volume 4, issue 1, , ISSN 21519617.

[15] Jabar H. Yousif, Information Technology Development, LAP LAMBERT Academic Publishing, Germany ISBN 9783844316704, 2011.
[16] Joachim Diederich , et.al, (2007). Ex-ray: Data mining and mental health, Applied Soft Computing 7 (2007) 923928. doi:10.1016/j.asoc.2006.04.007.

[17] Joachim Diederich , P. Yellowlees, A. Al-Ajmi(2003), Ex-Ray: Text Classification and the Assessment of Mental Health, Bruza, P., Thom, J. (Eds.), Proceedings of the Eighth Australian Document Computing Symposium, Canberra, Australia (15 December 2003) 75-76. Canberra: CSIRO Discovery Centre

http://joachimdiederich.com/assets/Ex-RayADCS03.pdf

[18] Lippman R. (1987), An introduction to computing with neural nets. IEEE Trans. ASSP Magazine 4, 4-22.

[19] Lodhi H., et.al,(2002). Text classification using string kernels. Journal of Machine Learning Research, 2, 419444. Paris: EC2 \& Cie. doi=10.1.1.130.2853

[20] Mabruk Ali Fekihal and Jabar H Yousif,(2012), "SelfOrganizing Map Approach for Identifying Mental Disorders", International Journal of Computer Applications 45(7):25-30, Published by Foundation of Computer Science, New York, USA

[21] Medical dictionary. http://www.medilexicon.com/medicaldictionary.php

[22] Teuvo Kohonen, ,(2001), Self-Organizing Maps, (3rd edition) Springer, ISBN 3540679219 .

[23] Zadeh, L.A. (2001). Applied Soft Computing. Applied Soft Computing 1, 1-2. 